\title{
Dynamics of quantized vortices in superfluid helium and rotating Bose-Einstein condensates
}

\author{
Makoto Tsubota, Kenichi Kasamatsu and Tsunehiko Araki \\ Department of Physics, Osaka City University, \\ Sumiyoshi-ku, Osaka 558-8585, Japan
}

(Dated: November 1, 2018)

\begin{abstract}
In this article, we review the research on the dynamics of quantized vortices in superfluid helium and rotating Bose-Einstein condensates with emphasis on the recent research done by our group. A quantized vortex is a topological defect that arises from the order parameter in Bose-Einstein condensation in which frictionless superfluid flows with quantized circulation around each vortex.

A quantized vortex was both predicted and discovered first in superfluid ${ }^{4} \mathrm{He}$ which was the first example of a Bose-Einstein condensate. Quantized vortices have been thoroughly studied in superfluid ${ }^{4} \mathrm{He}$; one of the principal problems was the superfluid turbulent state consisting of a tangle of quantized vortices in thermal counterflow. More recently, the interest has shifted to the nature of superfluid turbulence, apart from the case of counterflow. After briefly reviewing the earlier research and describing the current problems, we focus our review on superfluid turbulence and vortex filament dynamics. One of the important problems is how superfluid turbulence relates to classical turbulence. Superfluid turbulence was recently shown to have an energy spectrum consistent with the Kolmogorov law, which is an important statistical law in fully developed classical turbulence. We also describe the diffusion of an inhomogeneous vortex tangle with relation to the observed decay of vortices at very low temperatures where the normal fluid component is so negligible that the usual mutual friction does not work as a decay mechanism. In connection to the above discussion of groups of vortices, we describe the vortex states that appear in a rotating channel with counterflow. Rotational effects cause the vortices to form ordered arrays, whereas counterflow effects tend to cause disordered vortex tangles; the competition of these two effects makes a new state of "a polarized vortex tangle" which opens up superfluid phase diagrams as a new area of study.

In the specific field of atomic-gas Bose-Einstein condensation, we discuss recent numerical analysis of the Gross-Pitaevskii equation that describes the structure and dynamics of the order parameter. Consistent with observations, the simulated condensate starts an elliptic oscillation after the rotation is turned on, which induces the surface-mode excitations. The vortices develop from these surface excitations and then enter the bulk condensate, eventually forming a vortex lattice. When a condensate is held in a quadratic-plus-quartic combined potential, the fast rotation makes "a giant vortex" in which most vortices are absorbed into a central hole around which the superflow circulates.
\end{abstract}

PACS numbers:

\section{INTRODUCTION}

In Bose-Einstein condensations(BECs), a macroscopic fraction of bosons constitute a condensate wave function from which quantized vortices can form. A quantized vortex is a topological defect characteristic of a BoseEinstein condensate that has a quantized circulation of superflow and a thin vortex core of the order of the microscopic coherence length.

Quantized vortices were first predicted theoretically by Onsager 1] and Feynman 2]. Since their discovery in superflu id ${ }^{4} \mathrm{He}$ [3], research on quantized vortices has become one of the main subjects in low temperature physics [4]. On the other hand, the achievement of BoseEinstein condensation in dilute atomic gases [5] in 1995 has opened up a new research field, in which quantized vortices can be well controlled and visualized using new optical techniques. The study of quantized vortices has an important role in understanding the physics in both the traditional field of superfluid helium and the novel field of atomic-gas Bose-Einstein condensates (BECs).
Being motivated by the recent developments in these fields, our group has studied theoretically and numerically the dynamics of quantized vortices in superfluid ${ }^{4} \mathrm{He}$ and atomic BECs. The purpose of this article is to review the physics of quantized vortices in both systems with emphasis on the recent activities in our group [6].

Section summarizes the properties of quantized vortices and their differences from vortices in a classical fluid. Section IIII is concerned with the recent research of superfluid turbulence. Most of the early studies of superfluid turbulence were limited to the case of thermal counterflow, which has no analogy with classical turbulence. However, we also discuss recent experimental studies that help clarify the similarity between superfluid turbulence and classical turbulence. After describing the equation of motion of quantized vortex filaments, we discuss the energy spectrum of a vortex tangle in superfluid turbulence, the diffusion of an inhomogeneous vortex tangle, and a vortex tangle polarized by rotating the vessel. The vortex dynamics and vortex lattice formation of rotating BECs are described in SecIV Here we discuss the 
theoretical and numerical analysis of the problem. Recent experimental and numerical results show that elliptic deformation of the condensate and surface-mode excitations cause a vortex lattice to form. Section $\nabla$ is devoted to conclusions.

\section{WHAT IS A QUANTIZED VORTEX?}

Below a critical temperature in an ideal Bose gas, a finite fraction of the particles occupies the same singleparticle ground state and forms a BEC. When the particles have mutual interaction, single-particle states are no longer meaningful. However a condensate wave function $\Psi(\boldsymbol{r}, t)$ is still defined as the ensemble average of the quantum amplitude for removing a particle at position $\boldsymbol{r}$ from the condensate. When a BEC is held in an external potential $V(\boldsymbol{r})$, the dynamics of $\Psi(\boldsymbol{r}, t)$ is described by the Gross-Pitaevskii(GP) equation

$$
i \hbar \frac{\partial \Psi(\boldsymbol{r}, t)}{\partial t}=\left(-\frac{\hbar^{2}}{2 m} \nabla^{2}+V(\boldsymbol{r})+g|\Psi(\boldsymbol{r}, t)|^{2}\right) \Psi(\boldsymbol{r}, t),
$$

where $g=4 \pi \hbar^{2} m / a$ represents the strength of interaction characterized by the s-wave scattering length $a$ and $m$ is the mass of each particle. Writing $\Psi=|\Psi| \exp (i \theta)$, the squared amplitude $|\Psi|^{2}$ is the condensate density and the gradient of the phase $\theta$ gives the superfluid velocity $\boldsymbol{v}_{s}=(\hbar / m) \nabla \theta$, which is a frictionless flow of the condensate. As a result, the circulation of $\boldsymbol{v}_{s}$ around a closed path $\mathcal{C}$ in the fluid is quantized as

$$
\oint_{\mathcal{C}} d \boldsymbol{s} \cdot \boldsymbol{v}_{s}=\frac{\hbar}{m} \oint_{\mathcal{C}} d \boldsymbol{s} \cdot \nabla \theta=n \kappa \quad(n=0, \pm 1, \pm 2, \ldots),
$$

with the quantum of circulation $\kappa=h / m$. Such a vortex with a quantized circulation is called a quantized vortex; it occurs in superfluid ${ }^{4} \mathrm{He}$, superfluid ${ }^{3} \mathrm{He}$, and atomic BECs.

A quantized vortex is different from a vortex in a classical viscous fluid. First, the circulation is quantized, which is contrary to the classical vortex that can have any value of circulation. Second, a quantized vortex is a vortex of inviscid superflow. Thus, it cannot decay by the viscous diffusion of vorticity that occurs in the classical system. A quantized vortex can decay by shortening the length of the core through mutual friction with the normal fluid, by breaking into smaller and smaller vortex rings through reconnections and finally changing to some elementary excitations, and by transferring energy to smaller length scales through a Kelvin wave cascade, followed by acoustic emission. Third, the core of a quantized vortex is very thin, being the order of the coherence length defined by $\xi=\hbar /(\sqrt{2 m g}|\Psi|)$, which is submicron in an atomic-gas BEC and only a few angstroms in superfluid ${ }^{4} \mathrm{He}$. Because the vortex core is very thin and does not decay by diffusion, it is easy identify the position of a quantized vortex in the fluid. These properties make a quantized vortex more stable and definite than a classical vortex.

\section{DYNAMICS OF QUANTIZED VORTICES IN SUPERFLUID HELIUM}

\section{A. EARLY RESEARCH IN SUPERFLUID TURBULENCE AND VORTEX DYNAMICS}

Below the $\lambda$ temperature at about $2.2 \mathrm{~K}$ at vapor pressure, liquid ${ }^{4} \mathrm{He}$ enters the superfluid state called helium II. Although the condensate is depressed by interatomic interactions, this transition is microscopically caused by Bose-Einstein condensation. Helium II behaves like an irrotational ideal fluid, and its characteristic phenomena can be explained well by the two-fluid model 7 ]. The two fluid model states that superfluid ${ }^{4} \mathrm{He}$ is a mixture of inviscid superfluid and viscous normal fluid with the mixing ratio depending on temperature; the superfluid corresponds to the BEC [8] and the normal fluid has all of the thermal excitations of the system.

Most early experimental studies focused on thermal counterflow. When helium II is confined in a channel closed at one end with a heater, superfluid enters from the open end and flows toward the heater. On reaching the heater, normal fluid is created, which then flows back toward the open end. This situation is called thermal counterflow. It is laminar at low relative velocities, but when the relative velocity exceeds some critical velocity, the superflow becomes turbulent and has dissipation. The concept of superfluid turbulence was introduced by Feynman [2] who proposed that the superfluid turbulent state consists of a disordered set of quantized vortices, which is called a vortex tangle, that dissipates via mutual friction between the vortex cores and the normal flow. The mutual friction was introduced by Gorter and Mellink during their study of heat conduction through helium II in which they measured the relationship between temperature gradient and heat-current density [9]. Later, independently of Feynman's proposal, Hall and Vinen started the pioneering experimental works by making the simultaneous measurement of the temperature gradient and the second sound attenuation across counterflow. They discovered extra second sound attenuation and understood it in terms of the two fluid model with a generalized mutual friction [10]. After Feynman's proposal, Hall and Vinen confirmed that mutual friction occurs by observing the anisotropy of second sound attenuation in uniformly rotating helium II 11]. Later, Vinen investigated in detail the temperature gradient and the second sound attenuation across thermal counterflow to understand how the mutual friction changed when the heat current was suddenly changed. He found that the mutual friction came from rotational motion of superfluid, eventually establishing the picture of superfluid turbulent state consistent with Feynman's picture 12. The quantum of circulation was also measured by Vinen when he investigated the oscillation of a vibrating wire in rotating helium II 3]. There were many experimental studies of superfluid turbulence after Vinen's papers [13], chiefly on thermal counterflow. 
The nonlinear and nonlocal dynamics of vortices long delayed progress in further microscopic understanding of the vortex tangle. But Schwarz overcame these difficulties [14]. His most important contribution was his direct numerical simulation of vortex dynamics connected with dynamical scaling analysis, thus enabled others to calculate such physical quantities as the vortex line density, various anisotropic parameters, and the mutual friction force [14]. The observable quantities obtained with Schwarz's theory agreed well with the experimental results of the steady state of the vortex tangle. This research field pioneered by Schwarz has generated many new areas of study in vortex dynamics, such as the flow properties in channels 15, 16, 17, 18], sideband instability of Kelvin waves [19], vortex array in rotating superfluid [20], and vortex pinning [21, 22].

\section{B. RECENT STUDIES ON SUPERFLUID TURBULENCE}

Although our understanding of superfluid turbulence and vortex dynamics has made significant gains, the relation between superfluid turbulence and classical turbulence remains a major unsolved problem [23, 24]. Probably, this situation has arisen because most studies of superfluid turbulence have been devoted to thermal counterflow which has no analogy with classical turbulence.

We now discuss several relevant features of classical turbulence. In particular, we describe the finding that superfluid turbulence can give a clearer example of the inertial range than does classical turbulence. It has been known for over a hundred years that a classical viscous fluid becomes turbulent when the Reynolds number exceeds about 1000 25]. The Reynolds number is given by the ratio of the nonlinear inertial term to the viscous term in the Navier-Stokes equation; therefore, a large Reynolds number means that viscosity is less important compared with the inertial term. Apart from some boundary effects, at high Reynolds numbers, fully-developed, homogeneous turbulence follows some universal statistical laws. It turns out to be more helpful to consider energy and velocity in wave number $k$ space based on the spatial Fourier transform $\boldsymbol{v}(\boldsymbol{k})$ of the velocity field $\boldsymbol{v}(\boldsymbol{r})$. The important statistical law is represented in the energy spectrum $E(k)$, where $k=|\boldsymbol{k}|$ characterizes a length scale $k^{-1}$ and $E(k) d k$ is the average turbulent energy per unit mass in the range of the wave numbers $d k$. As an example, consider stationary grid turbulence in which a flow passing through a grid with the mesh size $D$ is close to being homogeneously turbulent far behind the grid. The behavior of $E(k)$ is classified into three ranges depending on the wave number. In the first energy-containing range, the energy is injected into the velocity field at the wave number $k \sim D^{-1}$, by a rate $\epsilon$ per unit mass. This energy flows into the second inertial range with higher wave number; this inertial range is characteristic of fully developed turbulence. Here the energy, not being dissipated, just flows toward the higher $k$ region by the energy flux $\epsilon$. The inertial range has no characteristic scale because it is sustained by the Richardson cascade process in which large eddies are continuously broken up self-similarly to smaller ones by a nonlinear interaction. Since the inertial range should be characterized only by the energy flux $\epsilon$ and the wave number $k$, the energy spectrum follows the well known Kolmogorov law

$$
E(k)=C_{K} \epsilon^{2 / 3} k^{-5 / 3} .
$$

Here, the Kolmogorov constant $C_{K}$ is found to be about 1.5 experimentally. The energy flowing from the inertial range into the third energy-dissipative range is finally dissipated by the dissipation rate $\epsilon$ at about the Kolmogorov wave number $k_{K}=\left(\epsilon / \nu^{3}\right)^{1 / 4}$ with the kinematic viscosity $\nu$. The important point is that the inertial range has a universal spectrum that is independent of the specific dissipative mechanism that operates in the energydissipative range. It is for this reason that superfluid turbulence is expected to follow the Kolmogorov law.

Recent experimental research studied superfluid turbulence not in thermal counterflow, thus finding support for the Kolmogorov law. Maurer and Tabeling measured local pressure fluctuations in helium flows driven by two counter-rotating disks in a range of temperature between 1.4 and $2.3 \mathrm{~K}$ and obtained the Kolmogorov spectrum above and below $T_{\lambda}$ 26]. A group at the University of Oregon reported in a series of papers [27, 28, 29, 30] the observed attenuation of second sound behind a grid that moved steadily through helium II at temperatures above $1 \mathrm{~K}$. Among these works, Stalp et al. measured the decay of grid turbulence in helium II and showed that the experimental results were consistent with a classical model of energy spectrum that included the Kolmogorov law [28]. Then, Vinen analyzed the similarity between superfluid turbulence and classical turbulence [24] and showed the importance of length scales for understanding the energy of the velocity field. For example, although superfluid turbulence is made of a tangle of quantized vortices, the situation depends on whether the length scale is larger or smaller than the vortex line spacing $\ell$. Furthermore, at relatively high temperatures, the normal fluid and the superfluid are coupled by mutual friction at all relevant scales larger than $\ell$, and hence the system follows the Kolmogorov law at these scales. This theory was shown to be consistent with the previous experimental results by Stalp et al., thus forming a consistent picture of superfluid turbulence with an appreciable component of normal fluid.

Then appears a very important question; how is the energy spectrum of superfluid turbulence at very low temperatures where the normal fluid component is negligible? This is significant in the following reasons. First, superflow guarantees that any dissipation could work only at some very small scale because of its inviscosity. This means the presence of the definite inertial range in which the energy is transferred from large scale to smaller scales by the Richardson cascade process. The resulting small 
vortices whose size becomes close to the coherence length would be unable to keep its vortex nature to change into some elementary excitations, or dissipate by acoustic emission [24], but the inertial range should be independent of such dissipative mechanisms. Second, superfluid turbulence is made of a tangle of quantized vortices. The inertial range of a classical fluid is believed to be sustained by the Richardson cascade, while the identification of each vortex in a turbulence is rather obscure. On the other hand, quantized vortices are definite and stable as described in Sec. II, so that the physical picture of the inertial range and the Richardson cascade could be much clearer than that in a classical turbulence. Therefore superfluid turbulence without the normal fluid component may give a typical and simple prototype of turbulence.

There are no experimental studies of the energy spectrum of superfluid turbulence at very low temperatures. Nevertheless, the two numerical works on this topic indicate that the Kolmogorov law also applies to superfluid turbulence at very low temperatures. Starting from a flow that mimicked a Taylor-Green vortex, Nore et al. studied the decaying turbulence by using the GP equation 31]. The energy spectrum succeeded in showing a transient Kolmogorov form over a range of wave numbers less than $\ell^{-1}$, but the acoustic emission is closely connected with the vortex dynamics and the situation is complicated. Our group studied the energy spectrum under the vortex filament formulation and found that the Kolmogorov law should apply to superfluids at very low temperatures 32. This formulation and the results will be described in the following subsections.

\section{DYNAMICS OF QUANTIZED VORTEX FILAMENTS}

All numerical calculations in this chapter were done under the vortex filament formulation. This subsection describes the equations of motion of quantized vortex filaments and the method of the numerical simulation. A vortex filament is an idealized model of rotational flow in which the vorticity is confined to a small core region around a one-dimensional line embedded in the threedimensional flow 33 . In the field of classical fluid dynamics, vortex filaments have been a useful model since the early 1980s for understanding the geometry and dynamics of a flow. However, in very recent years interest in this model has decreased because it is not capable of describing either the complicated turbulent structures of real systems or solutions of the Navier-Stokes equation [25]. But contrary to the situation in a classical fluid, vortex filaments are realistic in superfluids such as helium II, because here the cores are very thin and relatively stable. A quantized vortex filament may be likened to a "skeleton" of a vortex because the quantum mechanical constraint means that each vortex has the ground state circulation and a simple potential flow around its core. Our present main interest is to understand if a tan- gle of such quantized vortex filaments can still create the statistical law such as the Kolmogorov spectrum. This subsection is devoted to the description of the vortex filament formulation [14, 34], followed by a discussion of the energy spectrum in the next subsection.

The vortex filament formulation represents a quantized vortex as a filament passing through the fluid and having a definite direction corresponding to its vorticity. Except for the thin core region, the superflow velocity field has a classically well-defined meaning and can be described by ideal fluid dynamics. The velocity at a point $\boldsymbol{r}$ due to a filament is given by the Biot-Savart expression

$$
\boldsymbol{v}_{s}(\boldsymbol{r})=\frac{\kappa}{4 \pi} \int_{\mathcal{L}} \frac{\left(\boldsymbol{s}_{1}-\boldsymbol{r}\right) \times d \boldsymbol{s}_{1}}{\left|\boldsymbol{s}_{1}-\boldsymbol{r}\right|^{3}},
$$

where $\kappa$ is the quantum of circulation. The filament is represented by the parametric form $s=s(\xi, t)$ with the one-dimensional coordinate $\xi$ along the filament. The vector $s_{1}$ refers to a point on the filament and the integration is taken along the filament. Helmholtz's theorem for a perfect fluid states that the vortex moves with the superfluid velocity. Attempting to calculate the velocity $\boldsymbol{v}_{s}$ at a point $\boldsymbol{r}=\boldsymbol{s}$ on the filament makes the integral diverge as $\boldsymbol{s}_{1} \rightarrow \boldsymbol{s}$. To avoid this divergence, we separate the velocity $\dot{s}$ of the filament at the point $s$ into two components [14]:

$\dot{\boldsymbol{s}}=\frac{\kappa}{4 \pi} \boldsymbol{s}^{\prime} \times \boldsymbol{s}^{\prime \prime} \ln \left(\frac{2\left(\ell_{+} \ell_{-}\right)^{1 / 2}}{e^{1 / 4} a_{0}}\right)+\frac{\kappa}{4 \pi} \int_{\mathcal{L}}^{\prime} \frac{\left(\boldsymbol{s}_{1}-\boldsymbol{r}\right) \times d \boldsymbol{s}_{1}}{\left|\boldsymbol{s}_{1}-\boldsymbol{r}\right|^{3}}$.

The first term is the localized induction field arising from a curved line element acting on itself, and $\ell_{+}$and $\ell_{-}$ are the lengths of the two adjacent line elements, after discritization, that hold the point $s$ between them. The prime denotes differentiation with respect to the arc length $\xi$. The mutually perpendicular vectors $\boldsymbol{s}^{\prime}, \boldsymbol{s}^{\prime \prime}$ and $s^{\prime} \times s^{\prime \prime}$ point along the tangent, the principal normal and the binormal at the point $s$, respectively, and their magnitudes are $1, R^{-1}$, and $R^{-1}$ with the local radius $R$ of curvature. The parameter $a_{0}$ is a cutoff parameter equal to the core radius. Thus, the first term represents the tendency for the points to move the local point $s$ along the binormal direction with a velocity inversely proportional to $R$. The second term represents the nonlocal field obtained by integrating the integral of Eq. (4) along the rest of the filament.

The approximation that neglects the nonlocal terms and replacs Eq. (5) by $\dot{s}=\beta \boldsymbol{s}^{\prime} \times \boldsymbol{s}^{\prime \prime}$ is called the localized induction approximation (LIA). Here the coefficient $\beta$ is defined by $\beta=(\kappa / 4 \pi) \ln \left(c\langle R\rangle / a_{0}\right)$, where $c$ is a constant of order 1 and $\left(\ell_{+} \ell_{-}\right)^{1 / 2}$ is replaced by the mean radius of curvature $\langle R\rangle$ along the length of the filament. Most of Schwarz's numerical studies on vortex tangles used the LIA because this approximation can greatly reduce computation times. Although the method is effective for the analysis of dense tangles (due to cancellations between nonlocal contributions), it does not include the intervortex interaction properly. Because our present problems 
described in the following subsections need to take much account of the interaction, our numerical simulations are done by not the LIA but the fully Biot-Savart law of Eq. (5) .

A better understanding of vortices in a real system results when one includes the boundaries in the analyses. For this, the boundary-induced velocity field $\boldsymbol{v}_{s, b}$ is added to $\boldsymbol{v}_{s}$ so that the superflow can satisfy the boundary condition of an inviscid flow. If the boundaries are specular plane surfaces, $\boldsymbol{v}_{s, b}$ is just the field due to an image vortex made by reflecting the vortex into the plane and reversing its direction of vorticity. To allow for another, presently unspecified, applied field, we include $\boldsymbol{v}_{s, a}$. Hence, the total velocity $\dot{\boldsymbol{s}}_{0}$ of the vortex filament without dissipation is

$$
\begin{aligned}
\dot{\boldsymbol{s}}_{0}= & \frac{\kappa}{4 \pi} \boldsymbol{s}^{\prime} \times \boldsymbol{s}^{\prime \prime} \ln \left(\frac{2\left(\ell_{+} \ell_{-}\right)^{1 / 2}}{e^{1 / 4} a_{0}}\right) \\
& +\frac{\kappa}{4 \pi} \int_{\mathcal{L}}^{\prime} \frac{\left(\boldsymbol{s}_{1}-\boldsymbol{r}\right) \times d \boldsymbol{s}_{1}}{\left|\boldsymbol{s}_{1}-\boldsymbol{r}\right|^{3}}+\boldsymbol{v}_{s, b}(\boldsymbol{s})+\boldsymbol{v}_{s, a}(\boldsymbol{s}) .(6)
\end{aligned}
$$

At finite temperatures the mutual friction between the vortex core and the normal flow $\boldsymbol{v}_{n}$ is important. Including this term, the velocity of $s$ is given by

$$
\dot{\boldsymbol{s}}=\dot{\boldsymbol{s}}_{0}+\alpha \boldsymbol{s}^{\prime} \times\left(\boldsymbol{v}_{n}-\dot{\boldsymbol{s}}_{0}\right)-\alpha^{\prime} \boldsymbol{s}^{\prime} \times\left[\boldsymbol{s}^{\prime} \times\left(\boldsymbol{v}_{n}-\dot{\boldsymbol{s}}_{0}\right)\right],
$$

where $\alpha$ and $\alpha^{\prime}$ are the temperature-dependent friction coefficients, and $\dot{\boldsymbol{s}}_{0}$ is calculated from Eq.(6).

The method used in the numerical simulation is similar to that of Schwarz 14] and described in detail in our paper [34]. A vortex filament is represented by a single string of points with a distance $\Delta \xi$. The vortex configuration at a given time determines the velocity field in the fluid, thus moving the vortex filaments according to Eqs. (6) and (7). Both local and nonlocal terms are represented by means of line elements connecting two adjacent points. As the vortex configuration develops and, particularly, when two vortices approach each other, the distance between neighboring points can change. Then it is necessary to add or remove points properly to retain sufficient local resolution. Through the cascade process, a large vortex can break up through many reconnections, eventually becoming a vortex that is smaller than the space resolution $\Delta \xi$. The simulations cannot follow the dynamics beyond this point, so these vortices are eliminated numerically.

It is important to properly include vortex reconnection when simulating vortex dynamics. A numerical study of a classical fluid showed that the close interaction of two vortices leads to their reconnection, chiefly because of the viscous diffusion of the vorticity 35]. Schwarz assumed that two vortex filaments reconnect when they get close within a critical distance, and showed that the statistical quantities such as vortex line density were not sensitive to how to make reconnections. Even after the Schwarz's works, it was still unclear whether quantized vortices can actually reconnect or not. However Koplik and Levine solved directly the GP equation to show the two close quantized vortices reconnected even in an inviscid superfluid 36]. More recent simulations showed that reconnections were accompanied by acoustic emissions [37, 38]. As the vortex filament formulation cannot represent the reconnection process itself, we assume that two vortices reconnect when they approach within the space resolution $\Delta \xi$. The detail of this procedure is discussed in our paper [34].

\section{ENERGY SPECTRUM OF SUPERFLUID TURBULENCE}

To understand the energy spectrum and determine whether or not superfluids follow the Kolmogorov law at very low temperatures, one must calculate the energy spectrum. The energy spectrum is originally calculated by the Fourier transform of the fluid velocity $\boldsymbol{v}(\boldsymbol{r})$. The superfluid velocity $\boldsymbol{v}_{s}(\boldsymbol{r})$ is determined by the configuration of quantized vortices in our vortex filament formulation. Therefore, one can calculate the energy spectrum directly from the configuration of vortices. This is a big advantage of this formulation because it makes the numerical calculation of the spectrum less time-consuming than the method via $\boldsymbol{v}(\boldsymbol{r})$. Using the Fourier transform $\boldsymbol{v}_{s}(\boldsymbol{k})=(2 \pi)^{-3} \int d \boldsymbol{r} \boldsymbol{v}_{s}(\boldsymbol{r}) \exp (-i \boldsymbol{k} \cdot \boldsymbol{r})$ and Parseval's theorem $\int d \boldsymbol{k}\left|\boldsymbol{v}_{s}(\boldsymbol{k})\right|^{2}=(2 \pi)^{-3} \int d \boldsymbol{r}\left|\boldsymbol{v}_{s}(\boldsymbol{r})\right|^{2}$, the kinetic energy of the superfluid velocity per unit mass is

$$
E=\frac{1}{2} \int d \boldsymbol{r}\left|\boldsymbol{v}_{s}(\boldsymbol{r})\right|^{2}=\frac{(2 \pi)^{3}}{2} \int d \boldsymbol{k}\left|\boldsymbol{v}_{s}(\boldsymbol{k})\right|^{2} .
$$

The vorticity $\boldsymbol{\omega}(\boldsymbol{r})=\operatorname{rot}_{s}(\boldsymbol{r})$ is represented in Fourier space as $\boldsymbol{v}_{s}(\boldsymbol{k})=i \boldsymbol{k} \times \boldsymbol{\omega}(\boldsymbol{k}) /|\boldsymbol{k}|^{2}$, so that we have $E=\left((2 \pi)^{3} / 2\right) \int d \boldsymbol{k}|\boldsymbol{\omega}(\boldsymbol{k})|^{2} /|\boldsymbol{k}|^{2}$. The vorticity $\boldsymbol{\omega}(\boldsymbol{r})=$ $\kappa \int d \xi \boldsymbol{s}^{\prime}(\xi) \delta(\boldsymbol{s}(\xi)-\boldsymbol{r})$ in the vortex filament formulation is rewritten as $\boldsymbol{\omega}(\boldsymbol{k})=\left(\kappa /(2 \pi)^{2}\right) \int d \xi \boldsymbol{s}^{\prime}(\xi) \exp (-i \boldsymbol{s}(\xi)$. $\boldsymbol{k})$. Using the definition of the energy spectrum $E(k)$ from $E=\int_{0}^{\infty} d k E(k)$, these relations yield

$$
\begin{array}{r}
E(k)=\frac{\kappa^{2}}{2(2 \pi)^{3}} \int \frac{d \Omega_{k}}{|\boldsymbol{k}|^{2}} \iint d \xi_{1} d \xi_{2} \boldsymbol{s}^{\prime}\left(\xi_{1}\right) \cdot \boldsymbol{s}^{\prime}\left(\xi_{2}\right) \\
\times \exp \left(-i \boldsymbol{k} \cdot\left(\boldsymbol{s}\left(\xi_{1}\right)-\boldsymbol{s}\left(\xi_{2}\right)\right)\right),
\end{array}
$$

where $d \Omega_{k}=k^{2} \sin \theta_{k} d \theta_{k} d \phi_{k}$ is the volume element in spherical coordinates. This formula connects the energy spectrum directly with the vortex configuration.

Starting from the Taylor-Green vortex and following the vortex motion without the mutual friction, we obtained a roughly homogeneous and isotropic vortex tangle (Fig. 1) 32]. This is a decaying turbulence, being dissipated by the cutoff of the smallest vortices whose size is comparable to the space resolution $\Delta \xi=1.83 \times 10^{-2}$ $\mathrm{cm}$. At first, the energy spectrum has a large peak at the largest scale where the energy is concentrated, but the spectrum changes as the vortices become homogeneous and isotropic. The time dependence of the energy dissipation rate $\epsilon$ shows that $d \epsilon / d t$ becomes small after 


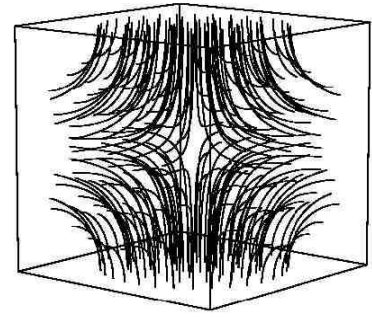

(a)

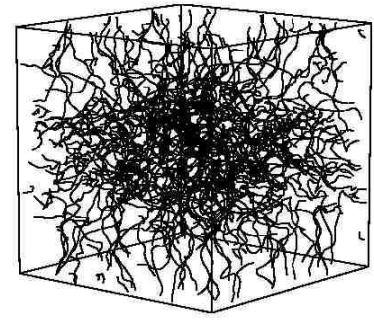

(c)

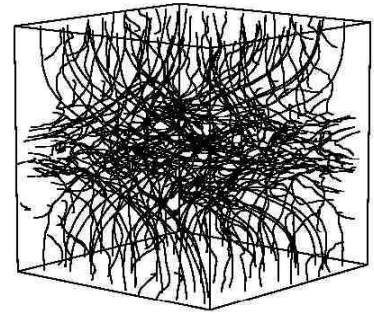

(b)

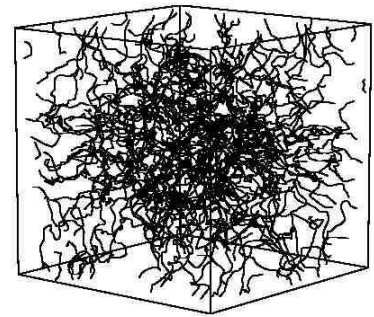

(d)
FIG. 1: Time development of the vortex tangle at $t=0 \mathrm{sec}$ (a), $30.0 \mathrm{sec}(\mathrm{b}), 50.0 \mathrm{sec}$ (c) and $70.0 \mathrm{sec}(\mathrm{d})$. The vortices are confined in a cube of size $D=1 \mathrm{~cm}$ and the calculation is made under the fully Biot-Savart law (Araki, Tsubota and Nemirovskii, Phys. Rev. Lett. 89,145301-2, 2002, reproduced w ith permission. Copyright(2002) by the American Physical Society).

about $70 \mathrm{sec}$ and artifacts of the initial state disappear 32. Similarly, the isotropic parameters introduced by Schwarz 14] indicate a nearly isotropic vortex tangle becomes after $70 \mathrm{sec}$. Figure 2 shows that the energy spectrum of the vortex tangle at $70 \mathrm{sec}$ agrees quantitatively with the Kolmogorov spectrum in the small $k$ region. The dissipative mechanism due to the cutoff works only at the largest wave number $k \sim 2 \pi / \Delta \xi=343 \mathrm{~cm}^{-1}$. However the energy spectrum at small $k$ region is determined by that dissipation rate. By monitoring the development of the vortex size distribution, such decay of a tangle is found to be sustained by the Richardson cascade process 34]. These results support the classical picture of the inertial range in superfluid turbulence at very low temperatures.

The power of the spectrum changes from $-5 / 3$ to -1 at about $k \sim 2 \pi / \ell$. The $k^{-1}$ spectrum comes from the contribution of the velocity near each single vortex. Our simulation of this $k^{-1}$ regime 39] indicates that the energy cascade process is due to the Kelvin wave cascade process. The simulations showed that when there is continuous excitation of small $k$ Kelvin waves along a single vortex and if a sink removes the energy at large wave numbers, then the nonlinear coupling between different modes leads to a net flow of energy from small to large wave numbers. This results in a simple steady spectrum of Kelvin waves that is insensitive to the strength and frequency of the excited drive.

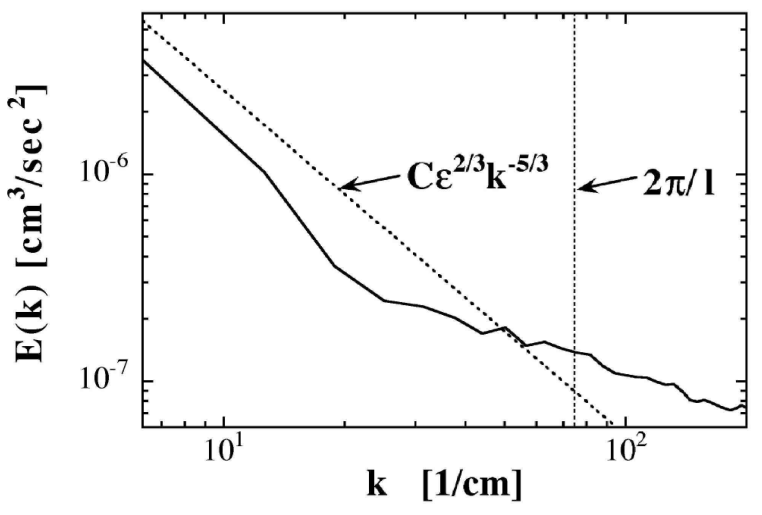

FIG. 2: Comparison of the energy spectrum (solid line) at $t=70 \mathrm{sec}$ with the Kolmogorov law $E(k)=C \epsilon^{2 / 3} k^{-5 / 3}$ (dotted line) with $C=C_{K}=1$ and $\epsilon=1.287 \times 10^{-6}$ $\mathrm{cm}^{2} / \mathrm{sec}^{3}$ law (Araki, Tsubota and Nemirovskii, Phys. Rev. Lett. 89, 145301-3, 2002, reproduced with permission. Copyright(2002) by the American Physical Society).

A specific picture of vortex tangles at very low temperatures has emerged from these studies. In the low $k$ regime, the vortex tangle has the inertial range in which the Kolmogorov spectrum is realized and the Richardson cascade process describes the energy flow to larger wave numbers; in this regime, superfluid turbulence mimics classical turbulence. In the regime with $k \geq 2 \pi / \ell$, the Kelvin wave cascade process becomes relevant to the transfer of energy to the high wave numbers above which some other mechanism dissipates the energy; this cascade process may be obscure in classical turbulence because the wave numbers become comparable to the vortex core sizes, but it is clearly seen in superfluid turbulence at very low temperatures.

\section{E. DIFFUSION OF AN INHOMOGENEOUS VORTEX TANGLE}

At finite temperatures, vortex tangles decay due to mutual friction. We ask here if vortex tangles decay at very low temperatures, where the normal fluid component is so negligible that the mutual friction does not work, and ask what mechanism removes energy from the tangle if it does decay. Experimentally, these questions are not easy to answer because counterflow and second sound techniques cannot work at such low temperatures. Nevertheless, Davis et al. created vortices in superfluid ${ }^{4} \mathrm{He}$ using a vibrating grid, and observed their temperature-independent decay below $T \sim 70 \mathrm{mK}$ by the trapping of negative ions at vortices [40]. The energy cascade process described in the previous subsection may be closely related to this decay, although an understanding of the behavior of an inhomogeneous vortex tangle is likely needed. Thus, we studied numerically the diffusion of a localized tangle [4]. We first prepared a localized 
vortex tangle. Then we followed its decay and diffusion under the fully Biot-Savart law in the absence of mutual friction.

The decay of a homogeneous vortex tangle is described by Vinen's equation $d L / d t=-\chi_{2}(\kappa / 2 \pi) L^{2}$ [42]. Here $L=(1 / \Lambda) \int d \xi$ is the vortex line density, where $\xi$ is the one-dimensional coordinate along the vortex filaments and the integral is taken along all vortices in the sample volume $\Lambda$. The parameter $\chi_{2}$ depends on temperature, being about 0.3 at zero temperature [34]. A simple generalization of this equation for an inhomogeneous system may be obtained by adding a diffusion term, so that we have the inhomogeneous Vinen's equaion

$$
\frac{L(\boldsymbol{r}, t)}{d t}=-\chi_{2} \frac{\kappa}{2 \pi} L(\boldsymbol{r}, t)^{2}+D \nabla^{2} L(\boldsymbol{r}, t),
$$

where $D$ is a diffusion constant. A comparison between the numerical solution of Eq. (10) and the above numerical simulation shows that $D=(0.1 \pm 0.05) \kappa$. Hence the diffusion of a tangle is relatively small; similar results were obtained by Barenghi and Samuels [43]. Even if a tangle is created by a vibrating grid, the diffusion is small and the tangle can remain localized in the neighborhood of the grid as far as there is no motion on a length scale larger than the line spacing. If motion on a larger scale is generated, then the diffusion can be enhanced.

\section{F. ROTATING SUPERFLUID TURBULENCE}

Rotating superfluid turbulence is interesting because the rotation can cause vortex ordering and thus lead to various vortex phases. This problem is different from that of superfluid turbulence at zero temperature, but closely related with the motivation becoming very important recently. Most configurations of quantized vortices which have been investigated in superfluid helium can be grouped into two types: ordered vortex arrays and disordered vortex tangles, and the former part of this article has been devoted to the disordered vortex tangle. For example, ordered arrays of vortices occur when superfluid helium is rotated with angular velocity $\Omega$ exceeding a certain critical value [4]. The resulting quantized vortices are aligned along the rotation axis and form an array whose areal number density is $2 \Omega / \kappa$, in agreement with a rule discovered by Feynman. Thus, an important question arose: what happens if vortices are created by both rotation and counterflow along the rotational axis? Only one experiment seems to address this issue 44]. In the experiment, Swanson, Barenghi, and Donnelly mounted a counterflow channel on a rotating cryostat, thus being able to create vortices by independent combination of rotation and counterflow. The vortex line density $L$ was determined from the secondsound attenuation across the channel. They found two critical velocities with $V_{c 1}<V_{c 2}$. At the lowest rotation velocities, when the counterflow velocity $V_{\mathrm{ns}}$ is less than $V_{c 1}$, a vortex array formed with a density in agreement with Feynman's rule. This critical value of $V_{c 1}$ was much less than that with no applied rotation. In addition, the measured $V_{c 1}$ was consistent with the onset of a vortex wave instability discovered experimentally by Cheng et al. 45] and explained by Glaberson et al. [46] as being caused by helical Kelvin waves along the vortex cores that are destabilized by the component of counterflow velocity along the vortices. This instability is usually called the Donnelly-Glaberson (DG) instability. The nature of the flow within this unstable regime and the cause of the second critical velocity $V_{c 2}$ is not clear.

To better understand these flow regimes, we simulated the original experiments of Swanson et al. 44], in which the superfluid flowed in a rotating cube, and found that the ordering of the vortex array was sensitive to the rotation velocity. In a rotating cube, the equation of motion of vortices is modified by two effects. The first effect is the force acting upon the vortex due to the rotation. According to Helmholtz's theorem, the generalized force acting upon the vortex is balanced by the Magnus force as $\rho_{\mathrm{s}} \kappa\left(\boldsymbol{s}^{\prime} \times \dot{\boldsymbol{s}}_{0}\right)=\delta F^{\prime} / \delta \boldsymbol{s}$, where $\rho_{\mathrm{s}}$ is the superfluid density, $F^{\prime}=F-\boldsymbol{\Omega} \cdot \boldsymbol{M}$ is the free energy of a system in the frame rotating with the angular velocity $\boldsymbol{\Omega}$, and $\boldsymbol{M}$ is the angular momentum. Taking the vector product with $\boldsymbol{s}^{\prime}$, we obtained the velocity $\dot{\boldsymbol{s}}_{0}$. The first term $F$ due to the kinetic energy of vortices gives the Biot -Savart law of Eq. (5). The second term $\boldsymbol{\Omega} \cdot \boldsymbol{M}$ leads to the velocity $\dot{\boldsymbol{s}}_{\text {rot }}$ of the vortex caused by the rotation. The second effect is the superflow induced by the rotating square crosssection. For a perfect fluid, we can use the analytical solution of the velocity $\boldsymbol{v}_{\text {cub }}$ inside a cube of size $D$ rotating with angular velocity $\boldsymbol{\Omega}=\Omega \hat{\boldsymbol{z}}$ [48]. By including both effects, we obtained the velocity $\dot{\boldsymbol{s}}_{0}$ in a rotating cube as the sum of $\boldsymbol{v}_{\text {cub }}$ and $\dot{\boldsymbol{s}}_{\text {rot }}$, the right-hand side of Eq. (5). In the numerical simulation, the sample was a cube of size $D=1.0 \mathrm{~cm}$. We used periodic boundary conditions along the rotating axis and rigid boundary conditions at the side-walls. Also, the counterflow was applied along the $z$-axis, the normal fluid was assumed to be at rest in the rotating frame, and, to make comparison with the experiment [4], we did the calculation for a temperature $T=1.6 \mathrm{~K}$. Hence, the calculation included mutual friction. Two quantities were introduced to characterize the vortex tangle. One is the vortex line density $L$, and the other is the polarization of the tangle which is defined as $\left\langle s_{z}^{\prime}\right\rangle=(1 / \Lambda L) \int d \xi \boldsymbol{s}^{\prime} \cdot \hat{\boldsymbol{z}}$. Thus $\left\langle s_{z}^{\prime}\right\rangle$ is unity for a perfect vortex array and zero for a randomly oriented tangle.

The numerical simulation began from a rotating vortex array with small random perturbations added to the vortices, then a counterflow velocity was applied. Figure 3 shows how 33 vortices, initially parallel to each other in a stable configuration at $\Omega=4.98 \times 10^{-2} \mathrm{rad} / \mathrm{sec}$, develop into a vortex tangle under $V_{\mathrm{ns}}=0.08 \mathrm{~cm} / \mathrm{sec}$. The parallel vortices become unstable and Kelvin waves grow by the DG instability. When the amplitude of the Kelvin waves becomes comparable to the average vortex separation, vortex reconnections take place and more vortex loops are created. These loops disturb the initial vortex 


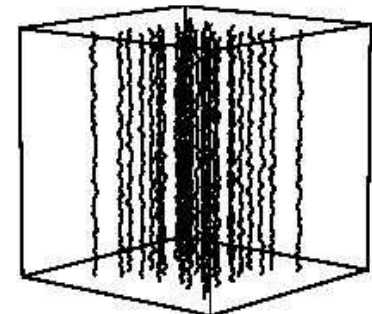

(a)

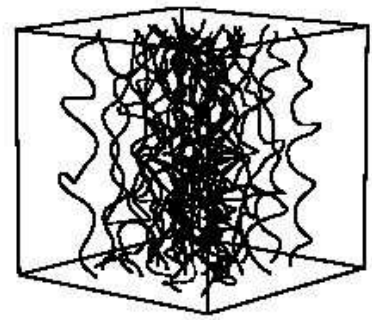

(c)

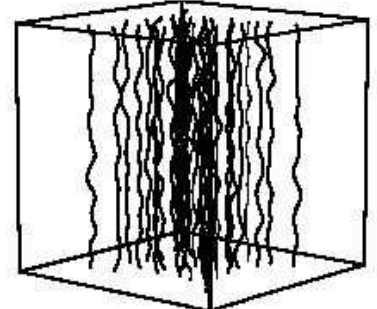

(b)

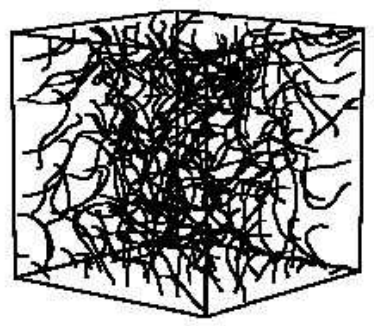

(d)
FIG. 3: Development from a vortex array to a polarized tangle at $T=1.6 \mathrm{~K}, \Omega=4.98 \times 10^{-2} \mathrm{rad} / \mathrm{sec}$ and $V_{n s}=0.08 \mathrm{~cm} / \mathrm{sec}$. Computed vortex tangle at the following times: $(\mathrm{a}): \mathrm{t}=0 \mathrm{sec}$; (b): 12sec; (c): $28 \mathrm{sec;} \mathrm{(d):} 160 \mathrm{sec}$ (Tsubota, Araki and Barenghi, Phys. Rev. Lett. 90, 145301-3, 2003, reproduced with permission. Copyright(2003) by the American Physical Society).

array, leading to an apparently random vortex tangle.

The results show that rotation tends to order the vortex tangle over that which would occur without rotation [14, 34]. The values of $L$ and $\left\langle s_{z}^{\prime}\right\rangle$ show that the vortex tangle evolves into a statistically saturated steady state. A vortex tangle created with only counterflow is sustained by the competition between the driving counterflow and the mutual friction [14], whereas in our case the steady state is achieved by balancing the effects of rotation, counterflow, and mutual friction. Figure 4 shows the dependence of $L$ and $\left\langle s_{z}^{\prime}\right\rangle$ on $V_{\mathrm{ns}}^{2}$ and $\Omega$ in each saturated state. It is evident that the polarization decreases with increasing counterflow velocity and increases with increasing rotation. This shows the competition between ordering caused by rotation and disordering caused by counterflow.

Recently Finne et al. used NMR to study turbulence of the B phase of superfluid ${ }^{3} \mathrm{He}$ and observed a sharp velocity-independent transition at a critical temperature between two regimes 49]. Regular behavior occurred at high temperature and turbulence occurred at low temperatures. They also found that the experimental results were consistent with numerical vortex dynamics simulations which was done by the method described here. This turbulence comes from intrinsic instability of vortices depending on temperature, being different from the DG instability.

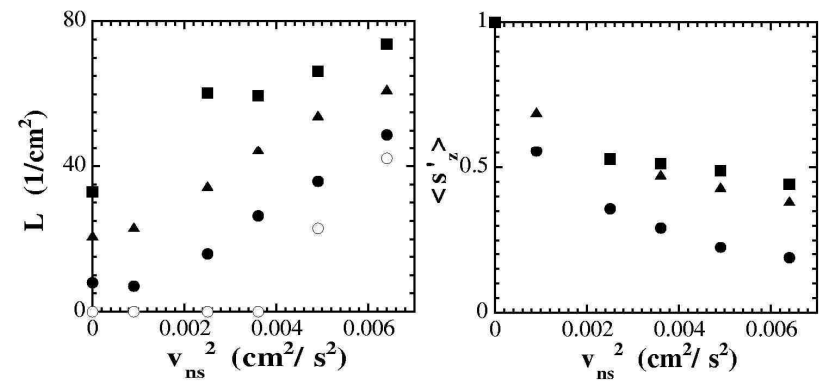

FIG. 4: Dependence of $L$ and $\left\langle s_{z}^{\prime}\right\rangle$ on $v_{n s}^{2}$ in the saturated state for $\Omega=0$ (white square), $\Omega=9.97 \times 10^{-3} \mathrm{rad} / \mathrm{sec}$ (black square), $\Omega=2.99 \times 10^{-2} \mathrm{rad} / \mathrm{sec}$ (circle) and $\Omega=4.98 \times 10^{-2}$ $\mathrm{rad} / \mathrm{sec}$ (triangle). The right figure lacks the data for $\Omega=0$ (Tsubota, Araki and Barenghi, Phys. Rev. Lett. 90, 1453013,2003 , reproduced with permission. Copyright(2003) by the American Physical Society).

\section{DYNAMICS IN ROTATING BOSE-EINSTEIN CONDENSATE}

\section{A. QUANTIZED VORTICES IN ALKALI-ATOMIC BOSE-EINSTEIN CONDENSATE}

The recent dramatic achievement of Bose-Einstein condensation in trapped alkali-atomic gases at ultra-low temperatures has stimulated intense experimental and theoretical activity [5]. Such atomic-gas Bose-Einstein condensates (BECs) differ fundamentally from liquid helium condensates in several ways. First, the condensates of alkali-atomic gases are dilute, having the mean particle density $n$ with $n|a|^{3} \ll 1$, so that the interatomic interaction can be accurately parametrized in terms of a scattering length $a$. As a result, at low temperatures, the GP equation (1) gives an extremely precise description of the atomic condensate and their dynamics. This situation differs from superfluid ${ }^{4} \mathrm{He}$, where the relatively high density and strong repulsive interactions complicate greatly the analytical treatments. Furthermore, because of the relatively strong interactions, the condensate fraction in bulk superfluid ${ }^{4} \mathrm{He}$ is only about $10 \%$ of the total particles, even at zero temperature. In contrast, almost all atoms participate in the condensate in an atomic-gas BEC. Second, a BEC in bulk helium has uniform density, whereas an atomic-gas BEC has nonuniform density due to the confinment by a trapping potential. In this section, we review the dynamics of quantized vortices in an atomic-gas BEC system.

From the above properties, the physics of quantized vortices has novel features. First, since the density is dilute, the relatively large coherence length $\xi$ makes it possible to directly visualize the quantized vortices by using optical techniques. Second, because the order of the coherence length $\xi$ is close to the size of the condensate, the vortex dynamics is closely connected with the collective motion of the condensate density itself. As 
described later, vortex nucleation of this system is related to an instability in the surface excitations of the condensate, which differs from that in superfluid helium system where the compressibility of the density is probably negligible. Finally, alkali atoms have internal degrees of freedom attributed to the hyperfine spin, so that their BECs can have multi-component order parameters if the spin degrees are available. Multicomponent BECs provide us with new possibilities to study unconventional vortex states that have been studied extensively in other fields of physics such as superfluid ${ }^{3} \mathrm{He}$, anisotropic superconductors, and cosmology.

Superfluids can support dissipationless flow, which is closely related to the existence of quantized vortices. Hence, the importance of studying a quantized vortex was recognized immediately after the first atomic-gas BEC was realized, in order to know whether or not the system becomes superfluid. The first experimental detection of a vortex involved a ${ }^{87} \mathrm{Rb}$ BEC containing two different internal hyperfine components [50]. The generation of a vortex was achieved by a phase engineering technique, in which interconversion between two components was controlled spatially and temporally by an external coupling field 51]. They then turned off the coupling, thus creating a vortex state consisting of one circulating component surrounding a nonrotating core of the other component. The ENS group observed the formation of single and multiple vortices in a single-component, ${ }^{87} \mathrm{Rb}$ elongated cigar-shape condensate [52]. The condensate was trapped in a static axisymmetric magnetic trap and a nonaxisymmetric attractive dipole potential created by a stirring laser beam. This combined potential produces a cigar-shaped harmonic trap with a slightly anisotropic transverse profile. By rotating the transverse anisotropy at a frequency $\Omega$, they could observe the formation of a vortex above a certain critical value of $\Omega$. A lattice consisting of more vortices appeared when $\Omega$ was increased further. In contrast to the indirect visualization methods used in superfluid helium systems, the quantized vortices were directly visualized as holes in the transverse density profile of the time-of-flight images. Later, groups at MIT [53], JILA [54], and Oxford 55] observed vortex lattices; the first two groups observed lattices that consisted of 100 or more vortices.

There are numerous theoretical studies of quantized vortices in atomic-gas BECs (see Ref. [56]). In this area, our group focused on the dynamical properties of quantized vortices. Based on the numerical simulation of the time-dependent GP equation, we revealed the marvelous nonlinear dynamics that a rotating BEC undergoes [57, 58, 59. In Sec. IVB we discuss the dynamics of vortex lattice formation in a rotating BEC [57, 59], motivated by the experimental observation of Ref. 60]. This work clarified for the first time the nonlinear dynamics of vortex lattice formation, in good agreement with the experimental results. In Sec. IVC we study the dynamics of a fast rotating condensate 58]. By combining an additional quartic potential with a harmonic potential, we find the new vortex states involving "a giant vortex", in which many vortices are absorbed in a single density hole.

\section{B. VORTEX LATTICE FORMATION IN A ROTATING BOSE-EINSTEIN CONDENSATE}

Madison et al. observed directly nonlinear dynamical phenomena such as vortex nucleation and lattice formation in a rotating condensate [60]. By suddenly turning on the rotation of the potential, the initially axisymmetric condensate made a collective quadrupole oscillation in which the condensate deformed elliptically. This oscillation continued for a few hundred milliseconds with a gradually decreasing amplitude. Then, the axial symmetry of the condensate suddenly reappeared and concurrently the vortices entered the condensate from its surface, eventually settling into a lattice configuration in the bulk. Such a direct observation of vortex nucleation and vortex lattice formation has never been done in superfluid helium. This observation motivated us to study theoretically the detailed dynamics of vortex lattice formation.

One motivation for studying a rotating, atomic-gas BEC is to determine whether the dynamical instability [61], the Landau instability [62], or both are responsible for vortex nucleation and lattice formation. The dynamical instability originates from the imaginary frequency of the excitation mode, giving rise to an exponential growth of the unstable mode even in the energy-conserving dynamics. The Landau instability occurs when the excitation spectrum has negative eigenvalues in the rotating frame and the system is subject to some energy dissipative mechanism. These two instabilities usually occur in different parameter regimes. Thus, one may ask which instability is important for actual vortex nucleation. In the experiments [52, 53, 60], the vortices nucleated most frequently when the rotation frequency $\Omega$ was near $0.7 \omega_{\perp}$, where $\omega_{\perp}$ is the transverse trapping frequency. The rotating potential in these experiments excites mainly the surface mode with the quantum number of angular momentum $l=2$ (quadrupole mode). When the interaction energy of the condensate is much larger than the energy of a harmonic potential, the dispersion relation for the surface mode is given by $\omega_{l}=\sqrt{l} \omega_{\perp}$ 63. In the rotating frame with frequency $\Omega$, the surfacemode frequency is shifted by $-l \Omega$. Hence, it is expected that the quadrupole mode $l=2$ is resonantly excited at $\Omega / \omega_{\perp}=\sqrt{2} / 2 \simeq 0.707$. Sinha and Castin showed that the rotating condensate without vorticity was dynamically unstable near the quadrupole resonance and proposed that this instability triggered vortex nucleation 61]. However, our later analysis reveals that both instabilities are necessary to explain the actual vortex formation.

We resolved these problems by numerically solving the 
GP equation which is an extension of Eq. (11) [57, [59]

$$
(i-\gamma) \hbar \frac{\partial \psi}{\partial t}=\left[-\frac{\hbar^{2}}{2 m} \nabla^{2}+V-\mu+g|\psi|^{2}-\Omega L_{z}\right] \psi .
$$

Here, the centrifugal term $-\Omega L_{z}=i \hbar \Omega\left(x \partial_{y}-y \partial_{x}\right)$ appears in a system rotating about the $z$-axis at a frequency $\Omega$. In this work, we assume translational symmetry along the $z$-axis, thus making the problem two-dimensional. Then the normalization of the wave function $\psi$ is taken as $\int d \mathbf{r}|\psi|^{2}=n_{2 \mathrm{D}}$ with the particle number per unit length along the $z$-axis. The trapping potential has the form

$$
V(\mathbf{r})=\frac{1}{2} m \omega_{\perp}^{2}\left\{\left(1+\epsilon_{x}\right) x^{2}+\left(1+\epsilon_{y}\right) y^{2}\right\},
$$

with the small anisotropy parameters $\epsilon_{x}$ and $\epsilon_{y}\left(\epsilon_{x} \neq \epsilon_{y}\right)$ ; this form describes approximately the rotating potential used in the ENS experiments [52, 60]. By introducing the scales characterizing the trapping potential $a_{h}=\sqrt{\hbar / 2 m \omega_{\perp}}$ for length and $\omega_{\perp}^{-1}$ for time, and replacing $\psi \rightarrow \sqrt{n_{2 \mathrm{D}}} \psi / a_{h}$, the coupling coefficient in the

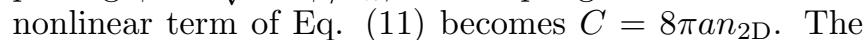
condition of Ref. [60], corresponding to $a=5.77 \mathrm{~nm}$, $N=3 \times 10^{5}, \omega_{z}=11.8 \times 2 \pi$ and $\lambda=\omega_{\perp} / \omega_{z}=9.2$, gives $C \simeq 500$. The term with $\gamma$ introduces dissipation, which is treated phenomenologically in the GP equation. This form of the dissipative equation follows the work of Choi et al. 64]; they studied the damping of the collective oscillation of a BEC and determined the value of $\gamma$ to be 0.03 by fitting their theoretical results with the MIT experiments [65]. Hence, we use $\gamma=0.03$ here. Because this dissipative term is much smaller than other terms in the GP equation, a small variation of $\gamma$ would not change the dynamics qualitatively but can only modify the relaxation time scale. This form can be derived from the formulation for a finite-temperature BEC developed by Zaremba et al. [66] under some approximations [59]. Gardiner et al. obtained the similar but different equation by another approach [67], applying it to the simulation of vortex lattice formation 68]. Because the time development of Eq. (11) does not conserve the norm of the wave function, we had to adjust the chemical potential $\mu$ at each time step to ensure normalization.

The simulations started from the stationary solution with uniform phase of Eq. (11) for a nonrotating trap $\left(\epsilon_{x}=\epsilon_{y}=0\right)$. The method for turning on a rotating drive followed the experimental procedure in Ref. [60]. The rotation with frequency $\Omega$ started at $t=0$, and the trap anisotoropy $\epsilon=\left\{\left(1+\epsilon_{x}\right)-\left(1+\epsilon_{y}\right)\right\} /\{(1+$ $\left.\left.\epsilon_{x}\right)+\left(1+\epsilon_{y}\right)\right\}$ was increased rapidly from zero to its final value 0.025 in $20 \mathrm{msec}$, keeping $\epsilon_{y}$ zero. First, we discuss the dynamics of the condensate with $C=500$ for $\Omega / \omega_{\perp}=0.7$. When the dissipation is free $(\gamma=0)$, the condensate undergoes a quadrupole oscillation, but it shows only the simple periodic oscillation in a sense of the Fermi-Pasta-Ulam recurrence [69] [see Fig. 6]. When the dissipation is present, this behavior is dramatically changed. The left column of Fig. 5 shows the time development of the condensate density $|\psi(x, y, t)|^{2}$. Initially, (a)
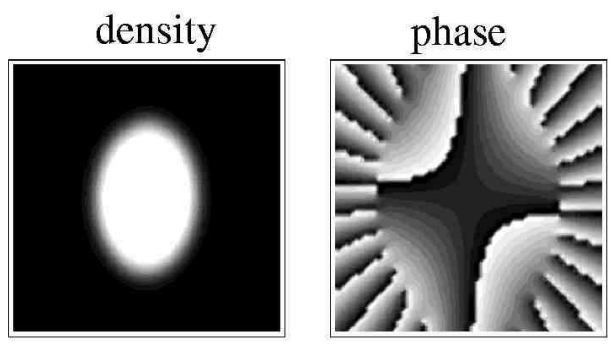

(b)
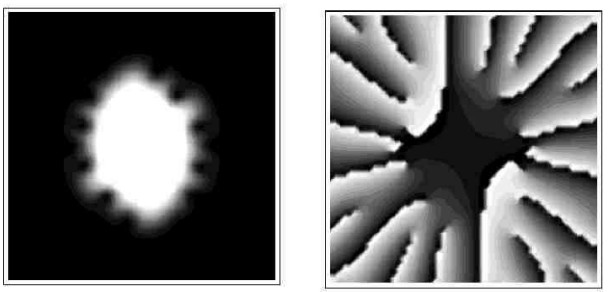

(c)
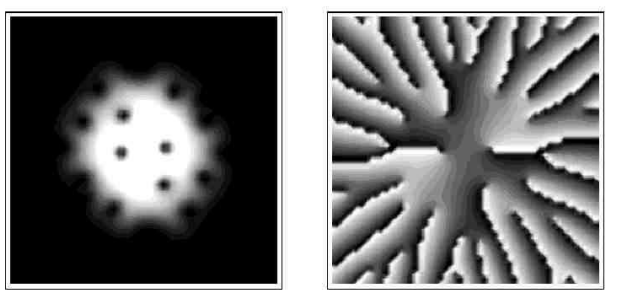

(d)
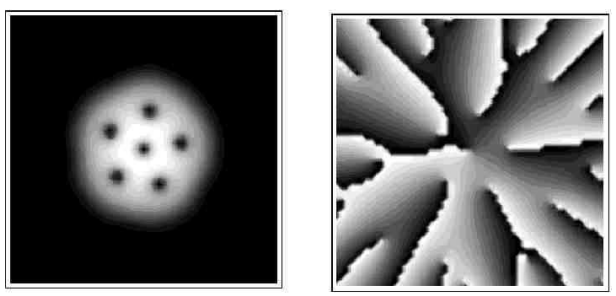

FIG. 5: Time development of the density (left column) and phase (right column) profile after the trapping potential begins to rotate suddenly with $\Omega=0.7 \omega_{\perp}$ for (a) $67 \mathrm{msec}$, (b) $360 \mathrm{msec}$, (c) $425 \mathrm{msec}$ and (d) $735 \mathrm{msec}$. The value of the phase varies continuously from 0 (black) to $2 \pi$ (white). There appear some lines where the phase changes discontinuously from black to white, corresponding to the branch cuts between the phase 0 and $2 \pi$. The apexes of these lines around which the value of the phase rotates continuously from 0 to $2 \pi$ represent the quantized vortices.

the condensate also undergoes a quadrupole oscillation, but the oscillation is damped because of the dissipation. After a few hundred milliseconds, the boundary surface of the condensate becomes unstable, generating surface ripples that propagate along the surface. The excitations are likely to occur on the surface whose curvature is low, i.e. parallel to the longer axis of the ellipse as shown in Fig. 5(b). Then the waves on the surface develop into the vortex cores, which enter the condensate. As the vortices penetrate inside the condensate, the axisymmetry of the condensate shape is recovered. As is known in the study of rotating superfluid helium [20], the rotating drive pulls vortices toward the rotation axis, whereas repulsive interactions between vortices tends to push them 


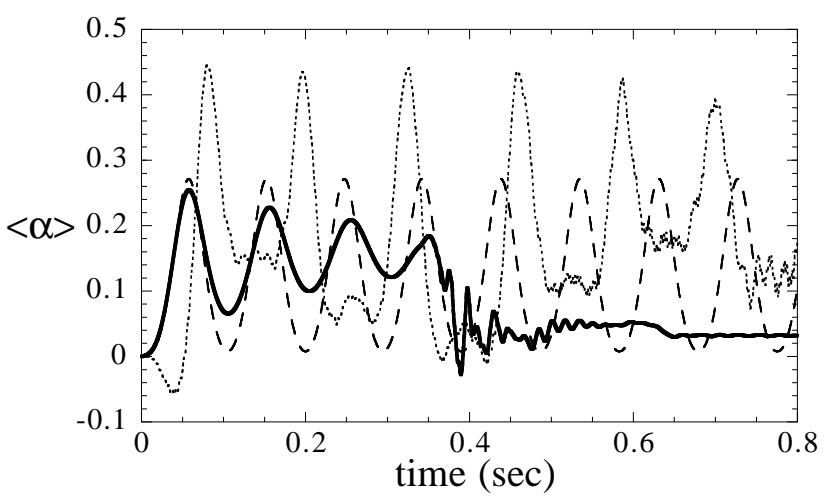

FIG. 6: Time evolution of the deformation parameter $\alpha$ for $\Omega / \omega_{\perp}=0.70$ with dissipation (bold-solid curve), for $\Omega / \omega_{\perp}=$ 0.70 without dissipation (dashed-curve) and for $\Omega / \omega_{\perp}=0.75$ without dissipation (dotted-curve).

apart; therefore, their competition yields a vortex lattice whose vortex density depends on the rotation frequency. In this simulation, six vortices form a vortex lattice. Figure 6 shows the time development of the deformation parameter defined as

$$
\alpha(t)=-\Omega \frac{\left\langle x^{2}\right\rangle-\left\langle y^{2}\right\rangle}{\left\langle x^{2}\right\rangle+\left\langle y^{2}\right\rangle},
$$

where $\langle A\rangle$ means $\int d x d y \psi^{*} A \psi$. Madison et al. observed that, after a rotation of $\Omega=0.7 \omega_{\perp}$ started suddenly, $\alpha$ oscillated during a few hundred milliseconds and then reduced abruptly to a value below 0.1 when vortices entered the condensate from its surface 60]. The evolution of $\alpha$ (bold-solid curve in Fig. 6) closely resembles Fig. 3 of Ref. 60], and our result is thus consistent with the experimental one.

The corresponding time development of the phase of $\psi(x, y, t)$ is shown in the right column of Fig. 5 . As soon as the rotation starts, the phase field inside the condensate takes the form of quadrupolar flow $\theta(x, y)=$ $\alpha(t) x y+$ const, and just outside the Thomas-Fermi boundary appear many proto-vortices; for example, Fig. 5 (a) shows about 20 proto-vortices. When the protovortices are on the outskirts of the condensate where the amplitude $|\psi(\mathbf{r}, t)|$ is almost negligible, they neither contribute to the energy nor the angular momentum of the system. Because they are invisible in the corresponding density profile of $\mathrm{F}$ ig. 5, they may be called "ghost vortices". The ghost vortices move toward the rotation axis, but their invasion into the condensate is prevented at the boundary surface within which the amplitude grows up. However, as the surface ripples are generated, the ghost vortices begin to penetrate the condensate. At this point, only some defects enter the bulk condensate because their further invasion costs energy. For example, Fig. 5(d) shows that six vortices enter the condensate and form a lattice, while the remaining vortices are repelled and escape to the outside of the Thomas-Fermi boundary.
The penetration of the ghost vortices into the condensate was accomplished with the help of the surface ripples, induced by the instabilities in the non-vortex state. One possible mechanism for the surface ripple excitation is the dynamical instability, which occurs without any dissipation. Sinha and Castin did a linear stability analysis of a rotating condensate, finding the growth of the fluctuation in the resonant range $0.72<\Omega / \omega_{\perp}<0.78$ 61. To confirm whether this instability leads to the vortex lattice formation, we calculated the dynamics for $\Omega / \omega_{\perp}=0.75$, and show the evolution of $\alpha$ in Fig. 6 . We can see that the perfect recurrence of the oscillation is broken. This is due to the dynamical instability, which causes the irreversible transfer of the quadrupole mode into the higher-energy excitation mode via the nonlinear mode coupling. However, although the surface ripples were generated, the ghost vortices never penetrated the inside of the condensate. Therefore, the dynamical instability alone cannot explain the vortex lattice formation. Actually, in the experiments, the vortices occurred at even lower off-resonant frequencies 52, 53, 55, 60], a finding that cannot be understood using the dynamical instability. The other instability is the Landau instability, which is applicable to the case with dissipation. The critical frequency of this instability can be expressed by the Landau criterion $\Omega_{c}=\min \left(\omega_{l} / l\right)$ for a rotating BEC [62]. The angular momentum number $l_{c}$ that yields $\Omega_{c}$ takes a value larger than 4 with the parameter used in experiments 62]. For $C=500$, we obtain $l_{c}=8$ and $\Omega_{c}=0.5 \omega_{\perp}$ from $\omega_{l}$ by numerically solving the Bogoliubov-De Genne equation. Thus, our simulation confirmed that this instability actually leads to the generation of vortices, where the surface ripples always evolved into the vortex cores and penetrated into the condensate.

In the range $0.72<\Omega / \omega_{\perp}<0.78$ where the dynamical instability is effective, the surface ripples are excited, but dissipation is needed for the vortices to penetrate into the condensate. Here the dissipation may originate in the thermal component, which should be almost negligible in the atomic gas experiments at very low temperatures. However, in the dynamical process of a condensate, there is a possibility that the thermal component will be produced under a strong perturbation. Hence the experimental results of Ref. [52, 53, 60] may be explained as follows. Consider a situation in which there is almost no dissipation at very low temperatures, namely, a case in which the Landau instability cannot occur. In a quadrupole resonance, however, the dynamical instability causes stochasticity in condensate oscillations, leading to the creation of the thermal component. Indeed, Hodby et al. has reported that the temperature of the system increases from $0.5 T_{c}$ to $0.8 T_{c}$ when the vortices form [55]. The time spent during this process is determined by the growth time of the dynamical instability, which is expected to be about 100 msec 61]. Thus, this oscillationcreated thermal component makes the dissipation effective, so the vortices can penetrate into the condensate via the Landau instability. To test this hypothesis we 
need to use analysis beyond the GP equation and thus we leave this issue for future study. In the other range of $\Omega$, a condensate makes only a stable quadrupole oscillation without dissipation. However, our results show that vortices may be generated at frequencies above $\Omega_{c}$ whenever finite dissipation occurs. Therefore, if one does an experiment in which the temperature is high enough to have effective dissipation, one should be able to observe the critical frequency given by the Landau criterion.

\section{GIANT VORTEX FORMATION IN A FAST ROTATING BOSE-EINSTEIN CONDENSATE}

In this subsection, we consider the dynamics of a rotating BEC trapped in a harmonic-plus-quartic potential [58]. The greater the rotation frequency, the greater the number of vortices in the lattice. In this study, we want to find out what happens when the size of the vortex cores becomes comparable with the vortex separation. In such a situation, the vortex lattice is predicted to melt via quantum fluctuation, leading to a quantum Hall-like state $70,71,72$. For a rotating condensate with frequency $\Omega$ in a harmonic trapping potential $(1 / 2) m \omega_{\perp}^{2} r^{2}$, the centrifugal potential cancels the confinement, thus preventing a BEC from rotating at $\Omega$ beyond $\omega_{\perp}$. Unfortunately, some authors have concluded that the study of a dense vortex lattice encounters significant difficulties arising from such a softening of the effective trap potential 73,74 . This difficulty can be avoided by introducing an additional quartic potential, so that the effective potential for the rotating condensate becomes

$$
V(r)=\frac{1}{2} m\left(\omega_{\perp}^{2}-\Omega^{2}\right) r^{2}+\frac{1}{4} k r^{4} .
$$

The quartic part of $V$ allows us to increase $\Omega$ above $\omega_{\perp}$, then the potential has a "Mexican hat" structure. Hence, we can expect the dynamics to be characteristic of the quadratic-plus-quartic potential for $\Omega>\omega_{\perp}$. As in the last subsection, we also consider a two-dimensional system subject to rotation $\boldsymbol{\Omega}=\Omega \hat{\mathbf{z}}$ by assuming translational symmetry along the $z$ axis. In the numerical simulation, the rotating drive is suddenly turned on by introducing a small anisotropy of the harmonic trap as $\left(\epsilon_{x} x^{2}+\epsilon_{y} y^{2}\right) / 4$. Finally, the small trap anisotropy $\left(\epsilon_{x}, \epsilon_{y}\right)$ is turned off adiabatically to obtain the axisymmetric final steady state.

Numerical simulations were done for several values of $\Omega$ at fixed $C=250$ and $k=1$. Figure 7 shows the time development of the density profiles for $\Omega / \omega_{\perp}=2.5$ and $\Omega / \omega_{\perp}=3.2$. As before, the ripples are excited on the condensate surface, and some ripples develop into vortex cores and penetrate into the condensate. For $\Omega / \omega_{\perp}=$ 2.5 , however, some penetrating vortices move toward the center, merging together to make a density hole at the center, around which some other vortices form a circular array. The phase profiles of the final steady state are shown in Fig. 7(d). Although some phase defects come (a)
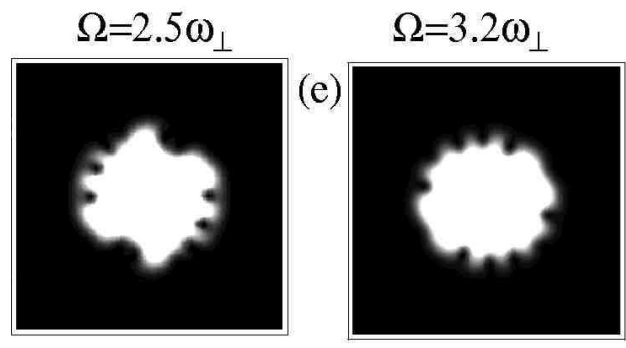

(b)

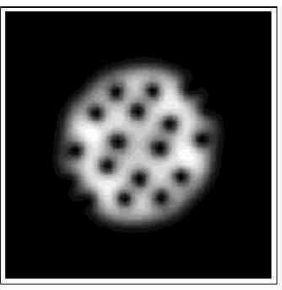

(f)

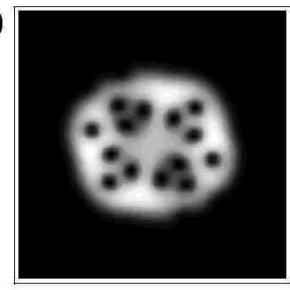

(c)

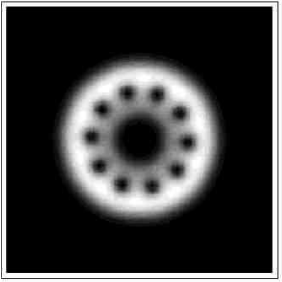

(g)

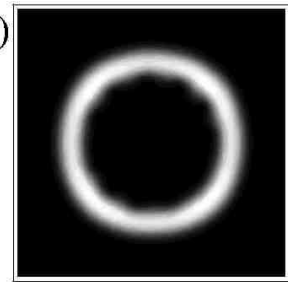

(d)

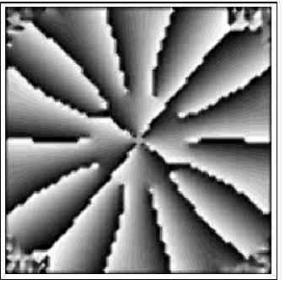

(h)

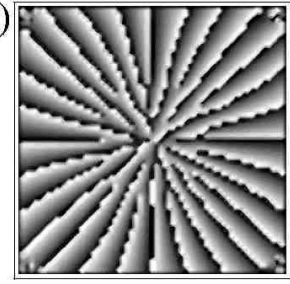

FIG. 7: Time development of the density profile for $\Omega=$ $2.5 \omega_{\perp}$ (left column) and $\Omega=3.2 \omega_{\perp}$ (right column) after the trapping potential begins to rotate suddenly. The time is (a) $30 \mathrm{msec}$, (b) $33 \mathrm{msec}$, (c) $300 \mathrm{msec}$ for the left column and (e) $20 \mathrm{msec}$, (f) $23 \mathrm{msec}$, (g) $200 \mathrm{msec}$ for the right column. The figure (d) and (h) shows the phase profile corresponding to Fig. (c) and (g), respectively.

very close to each other in the central hole, they never overlap.

As $\Omega$ is further increased, all vortices generated from the condensate surface are absorbed into the central density hole as shown in the right column of Fig. 7, where the central hole is composed of 24 singly-quantized vortices packed together. The packing is possible for high rotation frequencies $\left(\Omega>\omega_{\perp}\right)$ because the centrifugal force decreases the central condensate density, and thus packing the vortices together costs less energy. The minimum of Eq. (14) determines the radius of the ring condensate in Fig. $7(\mathrm{~g})$ as $R=\sqrt{m\left(\Omega^{2}-\omega_{\perp}^{2}\right) / k}$. Here we call a set of vortices such as that in Fig. 7(g) " a giant vortex" to indicate that a number of phase defects are contained in a single hole. Figures $7(\mathrm{~h})$ also shows that the phase singularities do not completely overlap. 


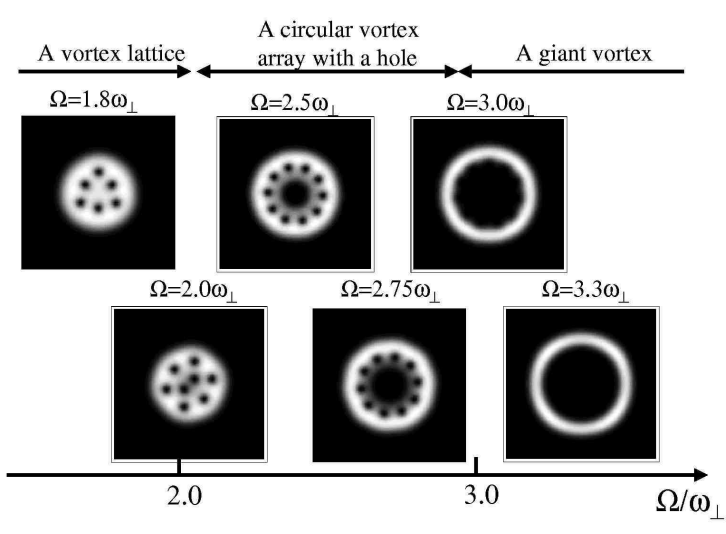

FIG. 8: The equilibrium vortex states obtained in the numerical simulation for $C=250$.

The obtained vortex states for the coupling coefficient $C=250$ are summarized in Fig. 8 as a function of a rotation frequency $\Omega$. For small $\Omega$, the equilibrium state is the vortex lattice state. As $\Omega$ increases, the vortices begin to merge in the central region. We find that two vortices merge at the center at $\Omega \simeq 2.2 \omega_{\perp}$. This is the onset of the appearance of a new vortex state consisting of a density hole and a circular array of vortices. Further increase of $\Omega$ (above 3.0) stabilized the giant vortex. Recently, Kavoulakis and Baym studied the vortex states in a fast rotating regime under the Thomas-Fermi approximation [75], which is effective only for the large interaction energy $C \geq 5000$. It is difficult to make the numerical simulation for such a strong interaction because the high rotation will generates an extremely large number of vortices, whose numerical description would need a very high spatial resolution. It is interesting to investigate the phase diagram of rich vortex states in a fast rotating BEC; Kavoulakis and Baym proposed a phase diagram with triple points between three distinct vortex states in the interaction strength versus rotation frequency plane 75.

Experimentally, this giant vortex has been created by Engels et al. 76. . The authors removed atoms of a rotating condensate from the central region, which resulted in the further increase of the angular momentum per particle. Eventually, about 60 vortices merged into a density hole, which had the long lifetime of many seconds. The combined harmonic-plus-quartic potential was created in ENS group recently by superimposing a blue detuned laser with the Gaussian profile [7]. They observed that, as the rotation frequency approached the frequency of the harmonic potential, the clear image of a vortex lattice disappeared, which suggested a transition into a new vortex phase.

\section{CONCLUSIONS}

In this review article, we discussed the dynamics of quantized vortices in superfluid helium and atomic-gas BECs. Analogous topological defects appear in almost all fields of physics, including superconductors, magnetic materials, and cosmology. In each such field, the defects play important roles in the physics. However, unlike most systems in which the physics of topological defects usually involve impurities, the vortices in superfluid helium and atomic BECs exist in very pure systems in which the proper physics of the topological defects themselves are relevant. This is the first reason why we are interested in vortices of these systems. The second reason is that quantum mechanical constraints make the vortices more stable and definite than those in classical media.

It has been about 40 years since a quantized vortex was discovered in superfluid helium. As described in this article, research on superfluid turbulence is about to reach a new stage regarding the similarity between superfluid turbulence and classical turbulence, a stage in which the nature of quantized vortices is crucial. In addition, the more recent realization of atomic-gas BECs have allowed us to visualize and control the dynamics of vortices, such that the quantum Hall state with a very high density of vortices may soon be reached [70, 71, 72]. Unconventional vortices in multicomponent BECs [8] is another area that will likely produce new and interesting results. Given the recent history of rapid developments in these fields, even more unexpected phenomena are likely to emerge in the future.

\section{Acknowledgments}

We are grateful for research collaboration with Sergey K. Nemirovskii, W.F.Vinen, Carlo F. Barenghi and Masahito Ueda. We thank Shigeo Kida, M.E. Brachet, P.V.E. McClintock, Yasuhide Fukumoto and Tetsuro Nikuni for useful discussions. M.T. acknowledges W.F.Vinen also for showing the detailed research history of superfluid turbulence. M.T. is grateful to the Japan Society for the Promotion of Science for financial support through the Japan-UK Scientific Cooperative program (Joint Research project) and through a Grant-in-Aid for Scientific Research (Grant no. 12640357 and 15340122).
[1] Onsager, L., 1949, Nuovo Cimento Suppl., 6, 249.

[2] Feynman, R.P. 1955, Progress in Low Temperature Physics Vol.1, C.J.Gorter (Ed.), North-Holland, Ameterdam, 17.

[3] Vinen, W.F., 1961, Proc. Roy. Soc., A260, 218.
[4] Donnelly, R.J. 1991, Quantized Vortices in Helium II, Cambridge University Press, Cambridge.

[5] Pethick, C.J. and Smith, H. 2002, Bose-Einstein Condensation in Dilute Gases,Cambridge University Press, Cambridge. 
[6] You can see the exciting movies on vortex dynamics in http://matter.sci.osaka-cu.ac.jp/bsr/top-e.html .

[7] Tisza, L., 1938, Nature, 141, 913; Landau, L., 1941, J. Phys. U.S.S.R., 5, 71.

[8] Exactly a superfluid component is not equivalent to a BEC. See Kobayashi, M., and Tsubota, M., 2002, Phys. Rev. B, 66, 174516.

[9] Gorter, C.J., and Mellink, J.H., 1949, Physica, 15, 285.

[10] Hall, H.E., and Vinen, W.F., 1955, Phil. Mag., 46, 546.

[11] Hall, H.E., and Vinen, W.F., 1956, Proc. Roy. Soc., A238, 204.

[12] Vinen, W.F., 1957, Proc. Roy. Soc., A240, 114 : 1957, Proc. Roy. Soc., A240, 128.

[13] Tough, J.T. 1982, Progress in Low Temperature Physics Vol.VIII, D.F.Brewer (Ed.), North-Holland, Ameterdam, 134.

[14] Schwarz, K.W., 1985, Phys. Rev., B31, 5782; 1988, Phys. Rev., B38, 2398.

[15] Samuels, D.C., 1992, Phys. Rev., B46, 11714; 1993, Phys. Rev., B47, 1107.

[16] Barenghi, C.F., Samuels, D.C., Bauer, G.H., and and Donnelly, R.J.,1997, Phys. Fluids, 9, 2631.

[17] Aarts, R.G.K.M., and de Waele, A.T.A.M., 1994, Phys. Rev., B50, 10069.

[18] Penz, H., Aarts, R., and de Waele, F., 1995, Phys. Rev., B51, 11973.

[19] Samuels, D.D., and Donnelly, R.J., 1990, Phys. Rev. Lett., 64, 1385.

[20] Tsubota, M., and Yoneda, H., 1995, J. Low Temp. Phys., 101,815

[21] Tsubota, M., and Maekawa, S., 1993, Phys. Rev., B47, 12040.

[22] Tsubota, M., 1994, Phys. Rev., B50, 579.

[23] Vinen, W.F., and Niemela, J.J., 2002, J. Low Temp. Phys., 128, 167.

[24] Vinen, W.F., 2000, Phys. Rev., B61, 1410.

[25] Frisch, U. 1995, Turbulence, Cambridge University Press, Cambridge.

[26] Maurer, J. and Tabeling, P., 1998, Europhys. Lett., 43, 29.

[27] Smith, M.R., Donnelly, R.J., Goldenfeld, N., and Vinen, W.F., 1993, Phys. Rev. Lett., 71, 2583.

[28] Stalp, S.R., Skrbek, L. and Donnelly, R.J., 1999, Phys. Rev. Lett., 82, 4831.

[29] Skrbek, L., Niemela, J.J., and Donnelly, R.J., 2000, Phys. Rev. Lett., 85, 2973.

[30] Stalp, S.R., Niemela, J.J., Vinen, W.F., and Donnelly, R.J., 2002, Phys. Fluids, 14, 1377.

[31] Nore, C., Abid, M., and Brachet, M.E., 1997, Phys. Rev. Lett., 78, 3896; 1997, Phys. Fluids, 9, 2644.

[32] Araki, T., Tsubota, M., Nemirovskii, S.K., 2002, Phys. Rev. Lett., 89, 145301.

[33] Samuels, D.C., 2000, Quantized Vortex Dynamics and Superfluid Turbulence, C.F.Barenghi, R.J.Donnelly, and W.F.Vinen(Ed.), Springer, Berlin, 97.

[34] Tsubota, M., Araki, T., and Nemirovskii, S.K., 2000, Phys. Rev., B62, 11751.

[35] Boratav, O.N., Pelz, R.B., and Zabusky, N.J., 1992, Phys. Fluids, A4, 581.

[36] Koplik, J., and Levine, H., 1993, Phys. Rev. Lett., 71, 1375.

[37] Leadbeater, M., Winiecki, T., Samuels, D.C., Barenghi, C.F., and Adams, C.S., 2001, Phys. Rev. Lett., 86, 1410.

[38] Ogawa, S., Tsubota, M., and Hattori, Y., 2002, J. Phys.
Soc. Jpn., 71, 813.

[39] Vinen, W.F., Tsubota, M, and Mitani, A., 2003, Phys. Rev. Lett., 91(in press).

[40] Davis, S. I., Hendry, P.C., McClintock, P.V.E., 2000, Physica B, 280, 43.

[41] Tsubota, M., Araki, T., and Vinen, W.F., 2003, Physica B, 329-333, 224.

[42] Vinen, W.F., 1957, Proc. Roy. Soc., A242, 493.

[43] Barenghi, C.F., and Samuels, D.C., 2002, Phys. Rev. Lett., 89, 155302.

[44] Swanson, C.E., Barenghi, C.F., and Donnelly, R.J., 1983, Phys. Rev. Lett., 50, 190.

[45] Cheng, D.K., Cromar, M.W., and Donnelly, R.J., 1973, Phys. Rev. Lett. 31, 433.

[46] Glaberson, W.I., Johnson, W.W., and Ostermeier, R.M., 1974, Phys. Rev. Lett. 33, 1197 ; Ostermeier, R.M., and Glaberson, W.I., 1975, J. Low Temp. Phys.21, 191.

[47] Tsubota, M., Araki, T., and Barenghi, C.F., 2003, Phys. Rev. Lett., 90, 205301.

[48] Milne-Thomson, L.M. 1968, Theoretical Hydrodynamics 5th Edition, Macmilan, London.

[49] Finne, A.P., Araki, T., Blaauwgeers, R., Eltsov, V.B., Kopnin, N.B., Krusius, M., Skrbek, L., Tsubota, M., and Volovik, G.E., 2003, Nature, 424,1022.

[50] Matthew, M. R., Anderson, B.P., Haljan, P.C., Hall, D.S., Wieman, C.E., and Cornell, E.A., 1999, Phys. Rev. Lett., 83, 2498.

[51] Williams, J.E., and Holland, M.J., 1999, Nature (London)., 401, 568.

[52] Madison, K.W., Chevy, F., Wohlleben, W., and Dalibard, J., 2000, Phys. Rev. Lett., 84, 806.

[53] Abo-Shaeer, J.R., Raman, C., Vogels, J.M., and Ketterle, W., 2001, Science., 292, 476.

[54] Haljan, P.C., Coddington, I., Engels, P., and Cornell, E.A., 2001, Phys. Rev. Lett., 87, 210403.

[55] Hodby, E., Hechenblaikner, G., Hopkins, S.A., Maragò, O.M., and Foot, C.J., 2002, Phys. Rev. Lett., 88, 010405.

[56] Fetter, A.L., and Svidzinsky A.A., 2001, J. Phys. B, 13, R135.

[57] Tsubota, M., Kasamatsu, K., and Ueda, M., 2002, Phys. Rev. A, 65, 023603.

[58] Kasamatsu, K., Tsubota, M., and Ueda, M., 2002, Phys. Rev. A, 66, 053606.

[59] Kasamatsu, K., Tsubota, M., and Ueda, M., 2003, Phys. Rev. A, 67, 033610.

[60] Madison, K.W., Chevy, F., Bretin, V., and Dalibard, J., 2001, Phys. Rev. Lett., 86, 4443.

[61] Sinha, S., and Castin, Y., 2001, Phys. Rev. Lett., 87, 190402.

[62] Dalfovo, F., and Stringari, S., 2001, Phys. Rev. A, 63, 011601(R).

[63] Stringari, S., 1996, Phys. Rev. Lett., 77, 2360.

[64] Choi, S., Morgan, S.A., and Burnett, K., 1998, Phys. Rev. A, 57, 4057.

[65] Mewes, M.-O., Andrews, M.R., Druten, N.J., Kurn, D.M., Durfee, D.S., Townsend, C.G., and Ketterle, W., ib 1996, Phys. Rev. Lett., 77, 988.

[66] Zaremba, E., Nikuni, and T., Griffin, A., 1999, J. Low Temp. Phys., 116, 277.@

[67] Gardiner, C.W., Anglin, J.R., and Fudge, T.I.A., 2002, J. Phys. B., 35, 1555.

[68] Penkwitt, A.A., Ballagh, R.J., and Gardiner, C.W., 2002, Phys. Rev. Lett., 89, 260402.

[69] Fermi, E., Pasta, J., and Ulam, S., 1965, Colleted Papers 
of Enrico Fermi (edited by E Segré, University of Chicago Press, Chicago, 1965).

[70] Ho, T.L., 2001, Phys. Rev. Lett., 87, 060403.

[71] Cooper, N.R., Wilkin, N.K., and Gunn, J.M.F., 2001, Phys. Rev. Lett., 87, 120405.

[72] Sinova, J., Hanna, C.B., and MacDonald, A.H., 2002, Phys. Rev. Lett., 89, 030403.

[73] Fetter, A.L., 2002, Phys. Rev. A, 64, 063608.

[74] Fischer, U.R., and Baym, G., 2003, Phys. Rev. Lett., 90, 140402.
[75] Kavoulakis, G.M., and Baym, G., 2003, New Jour. Phys. 5, 51.1 .

[76] Engels, P., Coddington, I., Haljan, P.C., Schweikhard, V., and Cornell, E.A., 2003, Phys. Rev. Lett., 90, 170405.

[77] Bretin, V., Stock, S., Seurin, Y., and Dalibard, J., cond-mat/0307464

[78] Kasamatsu, K., Tsubota, M., and Ueda, M., 2003, Phys. Rev. Lett., 91(in press). 